\title{
New evidence of SARS-CoV-2 transmission through the ocular surface
}

\author{
Huiling Qing ${ }^{1} \cdot$ Zhengwei Yang $^{1} \cdot$ Menghai Shi $^{1} \cdot$ Zongduan Zhang $^{2}$ (D)
}

Received: 4 April 2020 /Revised: 21 April 2020 / Accepted: 26 April 2020 / Published online: 4 May 2020

(C) Springer-Verlag GmbH Germany, part of Springer Nature 2020

\section{Dear Editor,}

The severe acute respiratory syndrome coronavirus 2 (SARS-CoV-2) causes systemic diseases and spreads worldwide, which seriously endangers patients' lives. By 2 April 2020 , confirmed cases were consecutively reported in majority of countries in the world. At this time, the rapid spread of the virus had caused 896,450 cases and 45,525 deaths globally [1]. So far, the large-scale epidemic has not been controlled, and the number of cases worldwide is still rising. Therefore, the routes of transmission have likely contributed considerably to the rapid spread of the 2019 novel coronavirus disease (COVID-19). It has been proven that the respiratory droplets and direct contact are the major routes for COVID-19 infection [2]. However, the route of fecal-oral and aerosol transmission, including through the ocular surface, have not been totally determined. Cutting off the route of transmission is one of the most important measures in the prevention and control of infectious diseases. Whether the ocular surface is a route of transmission remains to be proved.

In a retrospective study, the researchers included 1099 patients with laboratory-confirmed COVID-19 from 552 hospitals in China [2]. The results revealed that conjunctival congestion accounted for $0.8 \%$ (9/1099), which suggested the potential route of SARS-COV-2 transmission through the ocular surface. Conjunctivitis caused by SARS-COV-2 can be an early sign. SARS-CoV-2 could infect other persons without protection during the treatment.

In a prospective study, the investigators evaluated whether the novel coronavirus presented in tears and conjunctival se-

Huiling Qing and Zhengwei Yang contributed equally to this work.

Zongduan Zhang

zzduan@yeah.net

1 Department of ophthalmology, Henan Eye Hospital, Henan Eye Institute, Henan Provincial People's Hospital, Zhengzhou, China

2 Eye Hospital of Wenzhou Medical University, 270 Xueyuan Road, Wenzhou 325027, Zhejiang, China cretions of SARS-CoV-2-infected patients [3]. The samples were collected twice from each patient at an interval of 2 to 3 days. The results showed that of the 60 samples, tears and conjunctival secretions in two samples obtained from the only one patient with conjunctivitis yielded positive reversetranscription polymerase chain reaction (RT-PCR) results. They supposed that SARS-CoV-2 may exist in the conjunctival secretions and tears in novel coronavirus pneumonia (NCP) patients with conjunctivitis.

On 4 Mar 2020, Li and colleagues [4] observed that one NCP patient presented with conjunctivitis as the first symptom and another COVIN-19 case had conjunctivitis. The former patient had a history of closely contacting with confirmed COVID-19 patient. The SARS-CoV-2 RNA detection of nasopharynx swab was positive, but the conjunctival sac test was negative. The latter case presented a positive epidemiological history. The test results of both nasopharynx and conjunctival sac swabs were positive, which suggested that SARS-CoV-2 may transmit through the eyes and replicate in the ocular surface.

Another study showed that three rhesus macaques were inoculated with SARS-CoV-2. Two of them were randomly selected for ocular conjunctival inoculation, and the remaining one was inoculated via intratracheal route. The results revealed that virus could be detected in conjunctival swabs on the first day via ocular conjunctival route and then became undetectable indicating that the inoculated virus may transfer from conjunctiva to respiratory tract and other tissues. Viruses could be detected in nasal and throat swabs from three rhesus macaques 1 to 7 days after inoculation. Fourteen and 21 days after conjunctival inoculation with the virus, specific IgG antibodies against SARS-CoV-2 were detected in the rhesus macaques, indicating that the animal was indeed infected with SARS-CoV-2 [5]. The study results showed that conjunctiva is a route of SARS-CoV-2 transmission.

Yu Jun IS and colleagues reported that they collected 64 tear samples from 17 COVID-19 patients, but viral culture and RT-PCR detected no virus, which suggested a low risk of ocular transmission [6]. However, the samples were taken 
from first, second, and third week of initial symptoms. Patients with asymptomatic infections can carry the virus at early time. But the virus in tears may not be detected after symptoms appear since the non-stop secretion of tear and drainage through the lacrimal system could reduce virus load on ocular surface over time, which could further lead to negative detection.

Studies have found that SARS-CoV-2 can bind to human angiotensin converting enzyme 2 (ACE2) receptor, which is distributed among various tissue and cell types, including the conjunctiva [7]. SARS-CoV-2 has a similar receptor-binding domain structure to that of SARS-CoV, which suggests that the SARS-CoV-2 infection might have a similar pathogenesis to SARS-CoV infection [8]. ACE2 binding to the SARS-CoV2 is 10- to 20-fold higher than ACE2 binding to SARS-CoV S [9], demonstrating the potential for the SARS-CoV-2 transmission following exposure of unprotected eyes to this respiratory pathogen.

Based on current analysis and evidence, we argue that the potential ophthalmologic evidence of SARS-CoV-2 transmission through the ocular surface exists. Ocular surface infection is an important and potentially dangerous route of transmission, which is of great significance to protect clinicians, especially ophthalmologists, from infection.

However, many doctors have been infected by SARSCoV-2, of whom three ophthalmologists from the Wuhan Central Hospital died of COVID-19. These incidents have aroused great attention and enhanced the personal protection of ophthalmologists. Therefore, doctors are offered surgical masks, caps, goggles, and disposable gloves while receiving patients. Patients are asked to check the temperature and wear masks before entering the hospital. They also are requested to keep more than $1 \mathrm{~m}$ apart in hospital. The public is reminded to wear masks and pay attention to hand hygiene to prevent the transmission of virus. In addition, information sharing and action are the keys to constrain the pandemic.

\section{Compliance with ethical standards}

Conflict of interest The authors declare that they have no conflict of interest.

\section{References}

1. World Health Organization Novel Coronavirus (2019-nCoV). Situation report-73. https://www.who.int/docs/default-source/ coronaviruse/situation-reports/20200402-sitrep-73-covid-19.pdf? sfvrsn=5ae25bc7_4. Accessed 30 Mar 2020

2. W-j G, Ni Z-y HY et al (2020) Clinical characteristics of 2019 novel coronavirus infection in China. N Engl J Med. https://doi.org/10. 1056/NEJMoa2002032

3. Xia J, Tong J, Liu M, Shen Y, Guo D (2020) Evaluation of coronavirus in tears and conjunctival secretions of patients with SARSCoV-2 infection. J Med Virol. https://doi.org/10.1002/jmv.25725

4. Li X, Wang M, Dai J et al (2020) Novel coronavirus disease with conjunctivitis and conjunctivitis as first symptom: two cases report. Chin J Exp Ophthalmol. https://doi.org/10.3760/cma.j.issn.11598920200303-00133

5. Deng W, Bao L, Gao H, et al (2020) Rhesus macaques can be effectively infected with SARS-CoV-2 via ocular conjunctival route. https://www.biorxiv.org/content/10.1101/2020.03.13.990036v1. Accessed 30 Mar 2020

6. Yu Jun IS, Anderson DE, Zheng Kang AE et al (2020) Assessing viral shedding and infectivity of tears in coronavirus disease 2019 (COVID-19) patients. Ophthalmology [Article in press]. https://doi. org/10.1016/j.ophtha.2020.03.026

7. Zhou P, Yang XL, Wang XG et al (2020) A pneumonia outbreak associated with a new coronavirus of probable bat origin. Nature 579(7798):270-273

8. Lu R, Zhao X, Li J et al (2020) Genomic characterisation and epidemiology of 2019 novel coronavirus: implications for virus origins and receptor binding. Lancet 395(10224):565-574

9. Wrapp D, Wang N, Corbett KS et al (2020) Cryo-EM structure of the 2019-nCoV spike in the prefusion conformation. Science 367(6483): $1260-1263$

Publisher's note Springer Nature remains neutral with regard to jurisdictional claims in published maps and institutional affiliations. 